\title{
Paragonimiasis Miyazakii Associated with Bilateral Pseudochylothorax
}

\author{
Yoshikazu Inoue, Tomoya Kawaguchi, Akira Yoshida, Hisato Harada, Hideki Hara, \\ Satoru Yamamoto* and Mitsunori SAKatani
}

\begin{abstract}
A 37-year-old man who suffered from bilateral pleural effusions, subcutaneous abdominal induration and blood eosinophilia, was admitted to our hospital. He had ingested raw crabs at a pub-restaurant before the onset of his symptoms. His pleural effusions were chyliform containing cholesterol crystals, and a high level of immunoglobulin $\mathbf{E}$ $(36,580 \mathrm{IU} / \mathrm{ml})$ and anti-Paragonimus miyazakii antibody were detected. He was effectively treated with praziquantel. This case suggests that paragonimiasis should be strongly suspected if blood eosinophilia, pseudochylothorax, and a high level of immunoglobulin $E$ in pleural effusion are detected.
\end{abstract}

(Internal Medicine 39: 579-582, 2000)

Key words: eosinophilia, immunoglobulin E, cholesterol crystal, larva migrans, praziquantel.

\section{Introduction}

The prevalence of parasitic diseases has become low in Japan as the living environment has modernized. Because of this, it is difficult for the inexperienced physician, especially in urban areas, to detect these diseases. Patients with paragonimiasis suffer from pleural effusions, pneumothorax, intrapulmonary lesions, etc. The observation that pleural effusion is common (40-78\%) in these diseases has not been emphasized in the literature $(1,2)$, and paragonimiasis can be mistreated as tuberculosis $(3,4)$. We report a case of paragonimiasis miyazakii with bilateral pseudochylothorax and blood eosinophilia. In our case, precise examinations of pleural effusions including immunoglobulin E (IgE) measurement were helpful to the diagnosis.

\section{Case Report}

A 37-year-old man, who was a current smoker and alcohol drinker, but had no family or past history of specific disorders, was referred to our hospital because of bilateral pleural effusions and blood eosinophilia in July 1998. During the summer of 1996, he was drunken and jestingly ate two raw freshwater crabs (potamon dehaani) in a pub-restaurant in Osaka, Japan. In February 1997, he suffered sudden left chest pain, and was admitted to a hospital because of left pleurisy and blood eosinophilia. Although a conclusive diagnosis was not made, his symptom spontaneously improved and he was discharged. However, he continued to suffer from general fatigue. In June 1998, he went on a picnic, and ingested a small raw crab with alcohol for fun at the seashore near an estuary, and in the following one month he suffered from severe general malaise and sweating at night. Bilateral pleural effusions were found by his doctor and he was referred to our hospital.

On admission, we found a subcutaneous induration $(5 \times 4$ $\mathrm{cm}$, elastic hard) at the right abdominal wall, which spontaneously disappeared 10 days later. The patient was obese (body weight $=85 \mathrm{~kg}$, height $=178 \mathrm{~cm}$ ); anemia, icterus, or surface lymph node swelling were not observed; respiratory and heart sound, and body temperature were normal. His chest radiographs revealed bilateral pleural effusions (Fig. 1) and chest CT scan detected no apparent abnormal shadow in his lungs or mediastinal lymph node swelling (Fig. 2). Blood laboratory test on admission revealed blood eosinophilia (white blood cells count; $9,100 / \mathrm{ml}$, eosinophil $20.8 \%$ ); elevated erythrocyte sedimentation rate $(40 \mathrm{~mm} / 1$ hour $)$; C-reactive protein $(0.7 \mathrm{mg} / \mathrm{ml})$; $\alpha_{1}$-globulin (3.1\%, total protein $7.5 \mathrm{~g} / \mathrm{dl}$ ); $\alpha_{2}$-globulin (9.9\%); $\gamma$-globulin (21.7\%); aspartate amino transferase (35 IU/l); alanine aminotransferase (55 IU/l); $\gamma$-glutamyl transpeptidase (76 IU/l); and triglyceride $(351 \mathrm{mg} / \mathrm{dl})$. Blood levels of total cholesterol $(213 \mathrm{mg} / \mathrm{dl})$ and immunoglobulin E $(341 \mathrm{mg} / \mathrm{dl})$ were within normal ranges. Results of urinalysis, sputum, stool examinations were normal. Parasitic egg in sputum or stool was not detected. A skin test of purified protein derivative of tuberculosis was positive $(12 \times 13 / 40 \times 32 \mathrm{~mm})$. Vital capacity was

From the Department of Internal Medicine, *Pathology Unit, Department of Clinical Laboratory National Kinki-Chuo Hospital for Chest Diseases, Osaka Received for publication September 30, 1999; Accepted for publication February 7, 2000

Reprint requests should be addressed to Dr. Yoshikazu Inoue, the Department of Internal Medicine, National Kinki-Chuo Hospital for Chest Diseases, 1180 Nagasone-cho, Sakai, Osaka 591-8555 


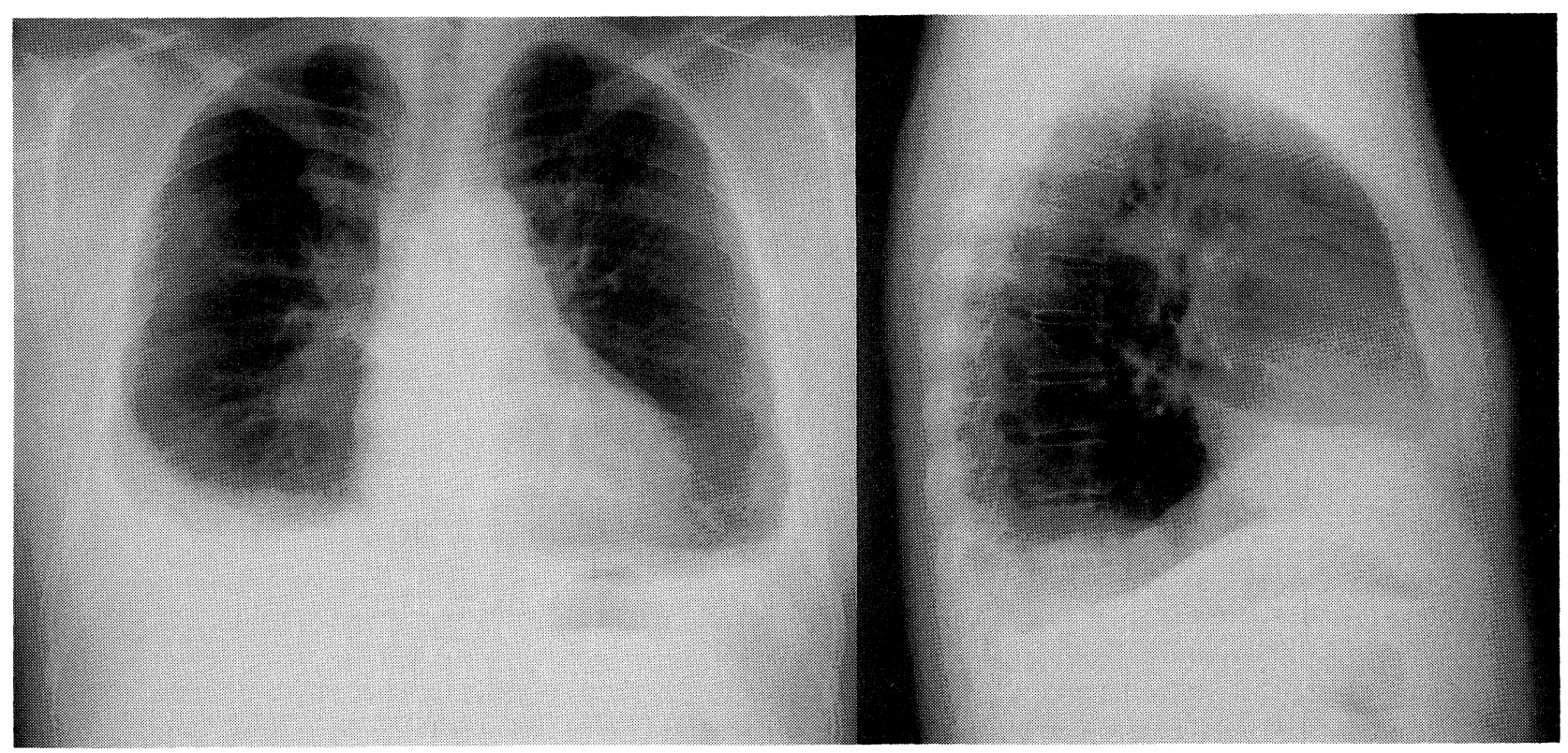

Figure 1. Chest radiograph on admission. Bilateral pleural effusions were found.

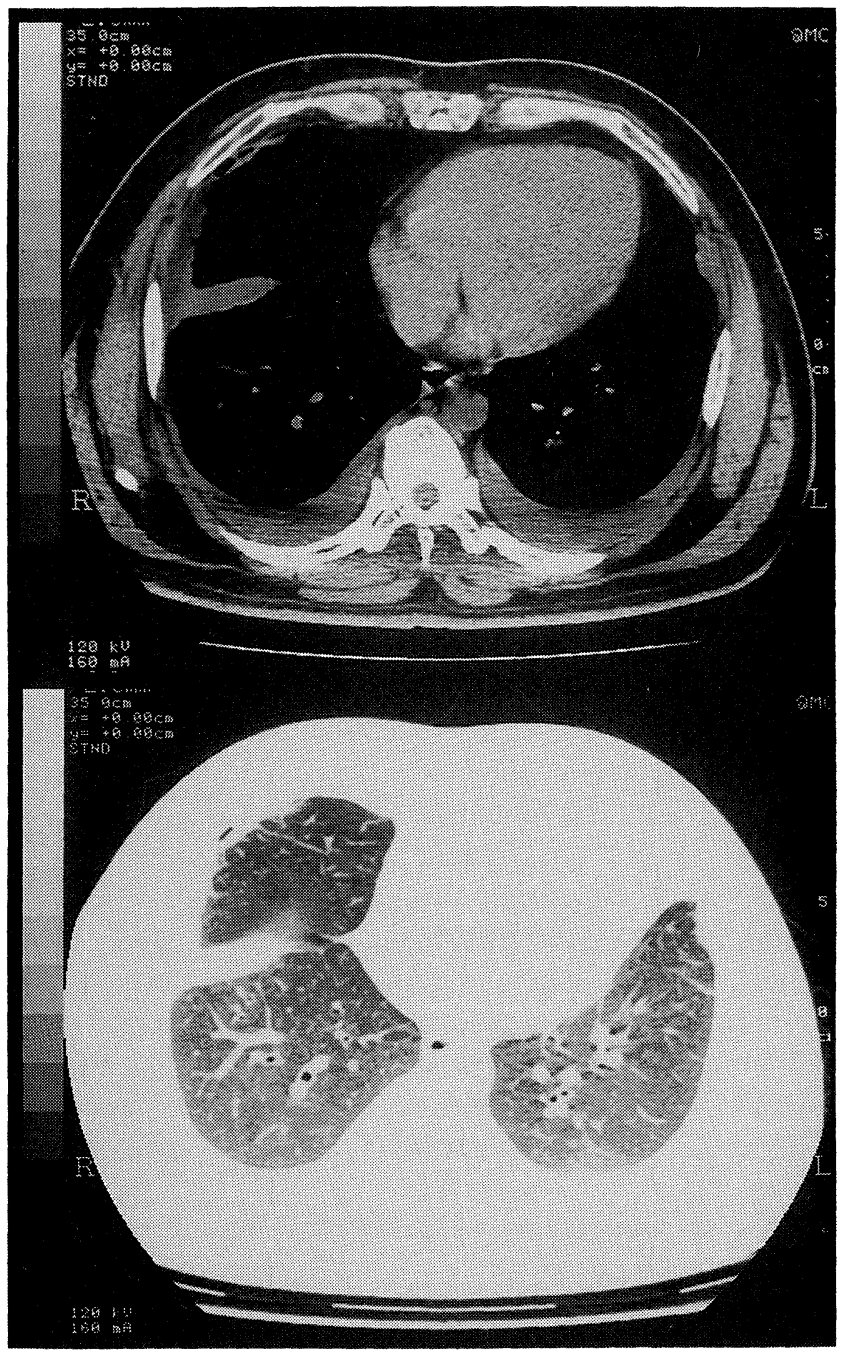

decreased to $62.6 \%$ of predicted. Abdominal CT scan detected only fatty liver. The subcutaneous induration in the right abdominal wall was not found, because the tumor was shrunken at the time of examination.

Thoracentesis and pleural biopsy were performed, but we could not detect a specific diagnostic finding from the biopsied pleura. Pleural effusions from both pleural cavities were turbid and milky yellow (Fig. 3, left). In order to distinguish chiliform from chylous effusion, we performed the following test. The effusion remained opaque after centrifugation but the turbidity cleared when ethyl ether was added. Microscopic finding revealed many large polyhedric crystals (Fig. 3; right), but no parasitic egg in the effusions. We concluded the effusion was chiliform (5). As shown in Table 1, the effusions were exudate with low glucose, low triglyceride, high lactate dehydrogenase, and high cholesterol level. IgE levels were extraordinarily high in both pleural effusions compared with serum IgE levels. When we performed thoracentesis again, appearance of right pleural effusion changed to bloody. In order to confirm the diagnosis, we measured serum anti-paragonimus antibody by enzyme immunoassay (SRL, Tokyo). The titer of antibody was borderline level $(32 x$; normal level $<32 \times)$. In order to obtain a specific diagnosis of paragonimiasis, we performed immunoelectrophoresis using his serum, pleural effusion, and antigens from Paragonimus westermani ( $P$. westermani) or $P$. miyazakii (kindly performed and advised by Professor Moriyasu Tsuji, Department of Tropical Diseases and

Figure 2. Chest CT scan. Bilateral pleural effusions were found. No abnormal finding in the lungs, or no lymph node swelling in mediastimun was found. 

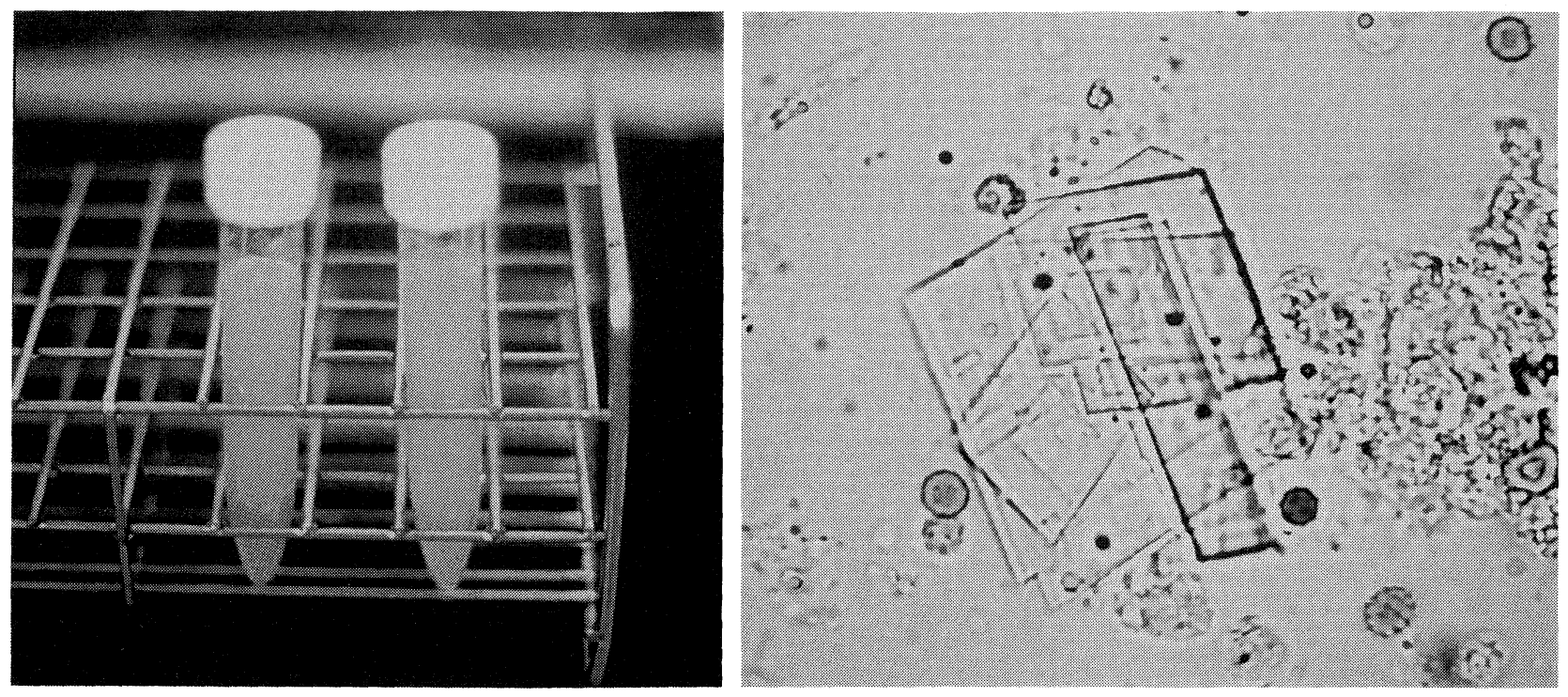

Figure 3. Turbid and milky yellow appearance of pleural effusions (left figure) and cholesterol crystals on microscopy $(\times 20$ objective; right figure).

Table 1. Analysis of Pleural Effusions

\begin{tabular}{lllll}
\hline & \multicolumn{3}{c}{ Pleural effusion } & \multirow{2}{*}{ Serum } \\
\cline { 2 - 4 } & Right (Aug. 4) & Right (Aug. 18) & Left (Aug. 18) & \\
\hline Appearance & turbid & bloody & turbid & \\
& milky yellow & & milky yellow & \\
Rivalta test & + & + & + & \\
Lymphocyte (\%) & 80 & 72 & 67 & \\
Neutrophil (\%) & 3 & 19 & 21 & \\
Eosinophil (\%) & 7 & 0 & 3 & 3.7 \\
Mesothelium/Macrophage (\%) & 10 & 7 & 9 & 286 \\
Total protein (g/dl) & 6.9 & 5.7 & 7.9 & \\
Glucose (mg/dl) & 14 & 38 & 0 & 448 \\
Lactate dehydrogenase (IU/l) & 1,698 & 3,002 & 4,360 & 165 \\
Cholesterol crystals & + & - & + & 378 \\
Egg & - & - & - & 1.9 \\
Triglyceride (mg/dl) & & 25 & 32 & \\
Total cholesterol (mg/dl) & & 150 & 329 & \\
Immunoglobulin E (IU/ml) & & 3,730 & 36,580 & \\
CEA (ng/ml) & 1.6 & 24.1 & NA & \\
Adenosine deaminase (IU/l) & NA & & 0.055 & \\
Hyaluronic acid (mg/ml) & & & & \\
\hline
\end{tabular}

CEA: Carcinoembryonic antigen, NA: not analyzed because the effusion was too turbid.

Parasitology, Kyorin University School of Medicine, Tokyo). We detected specific immunoprecipitant against $P$. miyazakii using pleural effusion but not serum. From these data, we concluded $P$. miyazakii caused his disease, and we treated the patient with praziquantel ( $3.3 \mathrm{~g} \times 2$ days). We also treated his hyperlipidemia with bezafibrate. After the treatments, his symp- toms and blood eosinophilia improved, and pleural effusions disappeared about two months later. The treatment using a regular dose of praziquantel was successful without adverse side effect, though slight dyspnea remained due to pleural adhesion. 


\section{Discussion}

Humans can acquire paragonimiasis by eating raw, undercooked, or pickled freshwater crustacean (crabs, crayfishes) which contain infective parasite forms (metacercariae). These forms enter the peritoneal cavity through the intestinal wall and migrate to the abdominal wall, diaphragm, and finally pleural cavity to the lungs, where they mature into adult worms and produce eggs (6).

In Japan, $P$. westermani, and $P$. miyazakii are important flukes. Compared with paragonimiasis westermani, paragonimiasis miyazakii does not cause cystic lesions in the lungs and, migrating between the pleural cavity and lungs, causes pleural diseases such as pneumothorax and pleural effusions. It is hard to detect eggs in sputum or stool. Humans are not comfortable hosts for P. miyazakii (2). A typical finding of pleural fluid from patients with paragonimiasis is exudate, containing low glucose levels, low $\mathrm{pH}$, high lactate dehydrogenase levels, and significant eosinophilia (5).

Yokogawa et al have reported that IgE levels in pleural fluid are higher than the simultaneous serum IgE levels in patients with pleural paragonimiasis (7). Ikeda et al demonstrated that paragonimiasis-specific IgE and IgG antibodies were much higher in pleural effusions than in sera (8). Whether the high pleural fluid IgE levels are specifically diagnostic of pleural Paragonimiasis remains to be determined (5). However, a high IgE level in pleural effusion and eosinophilia is strongly suggestive of this disease (9).

In long-standing pleural effusion, large amounts of cholesterol or lectin-globulin complexes can accumulate in the pleural fluid to produce a chyliform (not chylous) pleural effusion, pseudochylothorax (10). The turbulent pleural effusion from our patient was chyliform effusion containing many cholesterol crystals. Tuberculosis is by far the most frequent cause of pseudochylothorax (11). Though our patient had a positive skin test of tuberculosis, his chiliform effusion was not due to tuberculosis. Adenosine deaminase level in his pleural effusion was not high, and he was diagnosed by immunoelectrophoresis. Johnson and Johnson described 12 cases of chyliform pleural effusions secondary to paragonimiasis (1). Cholesterol crystals are found in many pleural effusions from patients with paragonimiasis (10). The mechanism of chiliform formation in paragonimiasis is not well understood.

The subcutaneous induration in the patient's abdominal wall disappeared spontaneously, and pleural effusion became bloody.
These findings might indicate larva migrans, although bloody pleural effusion could be a traumatic change after thoracentesis. Matumine et al reported only $3 \%$ of the patients with paragonimiasis miyazakii had subcutaneous lesions (2).

In conclusion, as indicated by this case, typical findings of pleural effusions including high levels of IgE and the appearance of chiliform are very diagnostic for paragonimiasis, as well as eosinophilia and eating history. Precise analysis of pleural effusion is essential before immunoelectrophoresis.

Acknowledgements: The authors thank Professor Moriyasu Tsuji (Department of Tropical Diseases and Parasitology, Kyorin University School of Medicine) for the immunological diagnosis by immunoelectrophoresis against paragonimus and pertinent discussions, Mr. Tomoaki Teramoto (National KinkiChuo Hospital for Chest Diseases) for technical assistance, Mr. Louis Garguilo and Naoko Inoue for reviewing the manuscript.

\section{References}

1) Johnson RJ, Johnson JR. Paragonimiasis in Indochinese refugees: roentgenographic findings with clinical correlations. Am Rev Respir Dis 128: 534-538, 1983.

2) Matumine $\mathrm{H}$, Araki $\mathrm{K}$. A case of paragonimiasis miyazakii appearing with bilateral pneumothorax, effusion and alternating chest pain -a review of 82 cases reported in Japan-. Nippon Naika Gakkai Zasshi 74: 597-605, 1985.

3) Fukunaga H, Baba M. A case of Paragonimus miyazakii infection in man showing bilateral pleural, interlobar and pericardial effusion with spontaneous pneumothorax. Nippon Kyobu Shikkan Gakkai Zasshi 15: 205209, 1977.

4) Kimura H, Kikui M, Tsuyuguchi I, Kishimoto S. A case of spontaneous remission of paragonimiasis miyazakii. Nippon Kyobu Shikkan Gakkai Zasshi 31: 1151-1156, 1993.

5) Light RW. Pleural diseases: Pleural effusion due to parasitic infection. 2nd ed. Lea \& Febiger, Philadelphia, 1990: 174-175.

6) Mahmoud AAF. Fishman's pulmonary diseases and disorders: Helminthic diseases of the lungs. Paragonimiasis. Fishman AP Ed., 3rd ed. McGraw-Hill, NewYork, 1998: 2411-2412.

7) Yokogawa M, Kojima S, Araki K, Tomioka H, Yoshida S. Immunoglobulin E: raised levels in sera and pleural exudates of patients with paragonimiasis. Am J Trop Med Hyg 25: 581-586, 1976.

8) Ikeda T, Oikawa Y, Owhashi M, Nawa Y. Parasite-specific IgE and IgG levels in the serum and pleural effusion of paragonimiasis westermani patients. Am J Trop Med Hyg 47: 104-107, 1992.

9) Iwahashi N, Suzuki F, Tamura S, et al. A case of paragonimiasis miyazakii with bilateral pleural and pericardial effusion. Nippon Kyobu Shikkan Gakkai Zasshi 29: 1047-1051, 1991.

10) Light RW. Pleural diseases: Chylothorax and pseudochylothorax. 2nd ed. Lea \& Febiger, Philadelphia, 1990: 269-281.

11) Hillerdal G. Chylothorax and pseudochylothorax. Eur Respir J 10: 11571162, 1997. 